\title{
Correction to: Perovskite Photovoltaics: From Laboratory to Industry
}

D. Forgacs, K. Wojciechowski and O. Malinkiewicz

\section{Correction to:}

Chapter 10 in: V. Petrova-Koch et al. (eds.), High-Efficient Low-Cost Photovoltaics, Springer Series in Optical Sciences 140, https://doi.org/10.1007/978-3-030-22864-4_10

The original version of the book was inadvertently published with typos in the abstract of Chapter 10, which have now been corrected. The book and chapter have been updated with the changes. 